\title{
Study of the anisotropy of the unresolved gamma-ray background
}

\author{
Michela Negro*t \\ Department of Physics, University of Torino and INFN/Torino \\ E-mail: michela.negrodto.infn.it
}

The extragalactic gamma-ray emission consists of two essential parts: resolved point-like or extended gamma-ray sources, and an isotropic component. When the former have been excluded (masked or subtracted), what remains is the latter component, called the Unresolved Gamma-Ray Background (UGRB), which, at a deeper level, is not truly isotropic and includes contributions from unresolved populations of sources.

The most recent measurement of the intensity energy spectrum of the UGRB was performed by Fermi Large Area Telescope (LAT) ${ }^{[2]}$ and it covers the energy range between 0.1 and $820 \mathrm{GeV}$. Models of known classes of gamma-ray emitters ${ }^{[3]}$ showed that the measured energy spectrum of the UGRB can be explained by the concomitant emission of unresolved blazars, star-forming and radio galaxies. However, the exact composition of the UGRB is still unknown. A measurement of the gamma-ray angular power spectrum can constrain the nature of the UGRB in a complementary way with respect to the intensity energy spectrum, since different populations of gamma-ray emitters are expected to induce different levels of anisotropies in the UGRB. Other observables that can be employed to a similar goal are the 1-point photon count probability distribution, the cross-correlation of the UGRB with galaxies (see e.g. [4]) or with galaxy clusters (see e.g. [6]), the cross-correlation with the weak lensing cosmic shear (see e.g. [7]) or with the gravitational lensing of the cosmic microwave background (see e.g. [5]).

In this analysis we study the UGRB anisotropy signal with eight years of Fermi-LAT Pass 8 data. Preliminary results are compatible with at least two classes of point-like sources contributing to the UGRB emission.

7th Fermi Symposium 2017

15-20 October 2017

Garmisch-Partenkirchen, Germany

\footnotetext{
* Speaker.

${ }^{\dagger}$ On behalf of the Fermi-LAT collaboration
} 


\section{Analysis Details}

The data selection applied for this analysis is designed to maximize the purity of the photon sample and the precision of its reconstructed arriving directions. For these reasons we use Pass 8 data of the ULTRACLEANVETO event class, and we reject the quartile of events with the worst Point Spread Function (PSF), that is all the events flagged as PSF0 type. This analysis considers about 8 years of data and covers the energy range from $158 \mathrm{MeV}$ up to $1 \mathrm{TeV}$. Ninety-five logarithmic "micro" bins subdivide the energy range and for each of them counts and exposure maps are computed. These are HEALPix ${ }^{[13]}$ maps of order $9\left(\mathrm{NSIDE}=512\right.$, pixel area $\approx 2.3 \times 10^{-4}$ $\mathrm{deg}^{2}$ ), which leads to 95 flux maps in $\mathrm{cm}^{-2} \mathrm{~s}^{-1} \mathrm{sr}^{-1}$ when dividing each count map with the corresponding exposure and for the pixel area. The flux maps are then summed up in order to have intensity maps in fourteen "macro" energy bins in the considered range. We do not compute flux maps directly on the "macro" bins in order to deal with the dependency on the energy of the exposure: the narrower the energy binning is, the more precise the interpolation of the exposure maps is, and consequently the computation of the mean exposure in each energy bin. The high statistics at low energies allows us to select only PSF3 event type below $1 \mathrm{GeV}$ (namely the quartile of events with the best values of containment angle), while above $1 \mathrm{GeV}$ we select the sample composed of the sum of PSF1, PSF2 and PSF3 flagged events.

Since we are interested in measuring and characterizing the flux fluctuations of the unresolved extragalactic component, the obtained flux maps are partially masked in a such a way that the majority of the Galactic emission and the contribution of the resolved sources are cut away.

The mask consists of a cut at $30 \mathrm{deg}$ in latitude around the Galactic plane and circular regions around each $3 \mathrm{FGL}^{[8]}$ source. In particular, given the containment angle at a certain energy $\left(P S F_{E}\right)$, the radii of the disks covering the sources vary logarithmically with the source integral flux from a minimum of $2 \times P S F_{E}$ to a maximum of $5 \times P S F_{E}$. In this way, the masking disks cover more the brightest sources and less the faintest ones, and shrink as the energy increases, reflecting the trend of the Fermi-LAT PSF. In addition, we mask the extended sources with larger radii $\left(10 \times P S F_{E}\right.$ for the LMC and CenA, and $5 \times P S F_{E}$ for all the others). Such a mask allows us to have statistics, especially at high energy, where the photon count is small.

In order to eliminate the residual Galactic contribution, we subtract the Galactic diffuse emission with the model described in [9]. Assuming that data in each pixel outside the mask are the sum of an isotropic emission component $C$ and the Galactic diffuse emission given by the model rescaled by a factor $N$, a maximum likelihood fit for Poissonian statistics over the micro binned data space returns the two parameters as a function of energy. The resulting normalized foreground maps are then subtracted from the data. The final masked maps are ready to be decomposed into spherical harmonics resulting eventually into Angular Power Spectra (APS), $C_{l}(l)$.

\section{Angular Power Spectrum}

In general, the autocorrelation APS of a full-sky intensity map is given by:

$$
C_{l}=\frac{1}{2 l+1} \sum_{m=-l}^{l}\left|a_{l m}\right|^{2}
$$


where

$$
a_{l m}=\int d \Omega_{n} F(\hat{n}) Y_{l m}^{*}(\hat{n})
$$

$\hat{n}$ being the direction on the sky, $Y_{l m}^{*}(\hat{n})$ the spherical harmonic functions evaluated at the direction $\hat{n}, F(\hat{n})$ the flux in the direction $\hat{n}$, and $l$ the multipole which is related to the angular scale $\theta$, since $l \approx \frac{\pi}{\theta}$. However, the measured APS is subject to a number of corrections which must be taken into account: 1) the sky is partially masked; 2) there is a contribution of the Poissonian white noise; 3) the measurement is affected by the instrument PSF and 4) the maps have finite resolution. By using PolSpice ${ }^{[12]}$ software to produce the APS, the effect of the mask is automatically taken into account by the algorithm, which also returns the covariance matrices. The Poissonian noise is a constant term, $C_{N}$, in the APS, and can be estimated as:

$$
C_{N}=\frac{\left.n_{\gamma, p i x}^{i} \widehat{/\left(A_{p i x}^{i}\right.}\right)^{2}}{\Omega_{p i x}}
$$

$n_{\gamma, p i x}^{i}$ being the photon counts in the unmasked pixels, $A_{\text {pix }}^{i}$ the exposure, $\Omega_{\text {pix }}$ the pixel solid angle. This number is computed for each "micro" energy bin and then summed in order to obtain the value for each "macro" energy bin.

The resolution of the maps and the effect of the PSF are taken into account respectively by the pixel window function, $W^{\text {pix }}(l)$, which is almost constant and equal to 1 for order 9 maps, and the beam window function, $W^{\text {beam }}(E, l)$, which is computed by the following formula for a given energy $\tilde{E}$ :

$$
W^{\text {beam }}(\tilde{E}, l)=2 \pi \int_{0}^{\pi} P_{l}(\cos \theta) P S F(\theta, \tilde{E}) \sin \theta d \theta
$$

where $P_{l}(\cos \theta)$ are the Legendre polynomials. Since the PSF is a function of the energy, it is necessary to average $W_{l}^{\text {beam }}(E)$ inside each energy bin weighted by the intensity energy spectrum of the UGRB, which is approximately a power-law with index $-2.3^{[2]}$.

So given the output from PolSpice, the APS of the signal is computed subtracting the white noise term and dividing by the window function squared:

$$
C_{l}^{\text {sig }}=\frac{C_{l}^{\text {PolSpice }}-C_{N}}{W_{E}(l)^{2}}
$$

where $W_{E}(l)=W^{p i x}(l) \cdot W_{E}^{\text {beam }}(l)$. Figure 1 shows the APS for three energy bins: they are compatible with a flat spectrum expected if the anisotropy signal is dominated by a population of unresolved point-like sources isotropically distributed in the sky. Under this hypothesis, we can fit the APS with a flat line in a specific range of multipoles, which returns the level of anisotropy, $C_{P}$, in each energy bin. Since, at low multipoles, residual background contamination might be present, while at high multipoles the correction for the PSF can be inaccurate, a proper definition of an energy dependent range of multipoles in which to perform the fit is crucial. To define the lower end of the fit, it is sufficient to compare the APS with and without foreground subtraction: this has shown that keeping $l_{\text {min }}=50$ in each energy bin is a conservative choice. To determine the upper end of the fit, a new quantity has been defined, which is sensitive both to the PSF improvement and the lack of statistics at increasing energies. This quantity, $R(\tilde{l})$, is given by the ratio between the absolute error of the APS at a given multipole, $\tilde{l}$, and the $C_{P}$ obtained by fitting the APS when the upper end of 
the multipole range is the same $\tilde{l}$. The final $l_{\max }$ is defined where $R(\tilde{l})$ becomes $>1$. The hatched regions in the three panels of figure 1, illustrate the range of multipoles in which the APS is fitted: you can notice how the upper end of the range moves to higher multipoles as the energy increases, due to the progressive improving of the PSF as a function of the energy. In order to stay away from any edge effect resulting from the APS calculation (the algorithm by default estimates the APS up to 3 (NSIDE) -1 , namely $l=1571$ for our order 9 maps), we do not consider multipoles higher than 1000 .

The anisotropy energy spectrum is then given by the obtained $C_{P}$ values as a function of the energy. A preliminary measurement can be found in figure 3(a). A fit of the measured APS under three spectral behaviors (power law, broken power, the sum of two power laws) shows that a single power law is disfavoured by the data. This kind of anisotropy spectrum can be produced by the concomitant emission from different classes of sources with different energy spectra, and a way to characterize this anisotropy signal, as suggested in Fornasa et al. ${ }^{[1]}$, is analyzing the crosscorrelation signal between different energy bins.
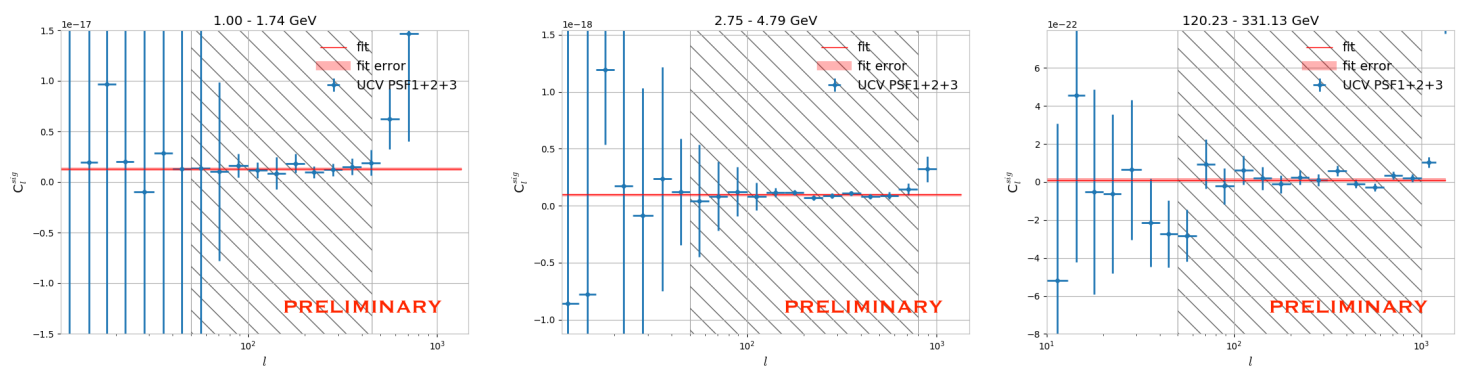

Figure 1: APS in three different energy bins: (left)1.-1.74, (center) 8.32-14.45, (right) 120.23-331.13 GeV. The red line is the fit of the APS, namely the $C_{P}$ value, and the range of multipoles involved in this fit is highlighted with the hatched region.

\section{Cross-correlation between energy bins}

The cross-APS between intensity maps in two different energy bins, $E_{i}$ and $E_{j}$, is computed as:

$$
C_{l}^{i j}=\frac{1}{2 l+1} \sum_{m=-l}^{l} a_{l m}^{i} a_{l m}^{j *}
$$

Similar to the auto-APS, the measured cross-APS is corrected for the PSF, but in this case the white noise term is zero, since the correlated maps are independent. So the cross-correlation signal is given by:

$$
C_{l}^{i j, s i g}=\frac{C_{l}^{i j, \text { PolSpice }}}{W_{E_{i}}(l) W_{E_{j}}(l)}
$$

Again we find flat cross-APS, and the level for each combination of correlated maps is $C_{P}^{i j}$. It is easy to demonstrate that $C_{P}^{i j}$ is predicted by the quantity $\sqrt{C_{P}^{i i}, C_{P}^{j j}}$ if the anisotropy signal is due to a single class of point-like sources, where $C_{P}^{i i}$ and $C_{P}^{j j}$ are the auto-correlation anisotropies in 
the energy bin $i$ and $j$ respectively. The ratio between the two quantities is called cross-correlation coefficient, $r_{i j}=\frac{C_{P}^{i j}}{\sqrt{C_{P}^{i i}, C_{P}^{j j}}}$, which could indicate that the signal is due to multiple populations of sources whenever it is below 1 . Figure 2 shows the results for the cross-correlation anisotropy energy spectra for two different energy bins: low energy bins clearly correlate with the nearby low energy bins and less with high energy bins, while the opposite behaviour can be observed for high energy bins. This could mean that the sources which contribute to the signal at low energy are not localized at the same position of those which contribute at high energy, hence there is more than one class of sources.

However, the physical interpretation of these results is not trivial. The detection efficiency of the instrument of point-like sources can play an important role, since in general, it is a function of the energy and the spectral index of the distribution of the source populations. The measurement of the auto- and cross-correlation anisotropy energy spectra is dependent on the catalog used to mask the resolved sources, which also gives a definition of what has to be considered "unresolved". Masking different source catalogs, which correspond to different detection efficiencies ${ }^{[10]}$, results in different measurements of anisotropy energy spectrum. It is possible to understand this statement by looking at the plot in figure 3(b), which illustrates the comparison between different anisotropy energy spectra of the UGRB obtained by masking different catalogs of sources: the 3FGL (pink and red points), the $3 \mathrm{FHL}^{[11]}$ (yellow points), a preliminary version of the 4FGL, and some "custom catalogs" built from data maps in 5 different energy bins. The latter case is the best choice for this analysis since it is possible to estimate the detection efficiency in energy bins and infer a more consistent interpretation from the data.

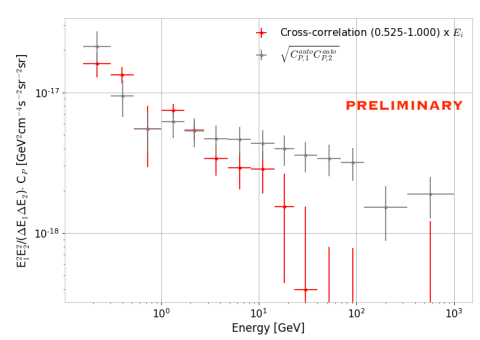

(a)

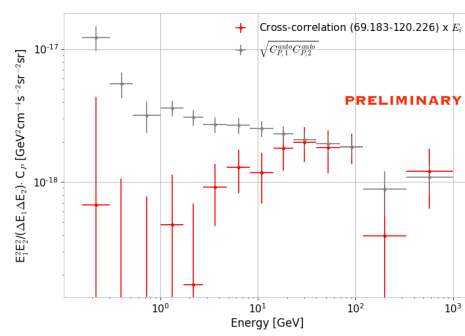

(b)

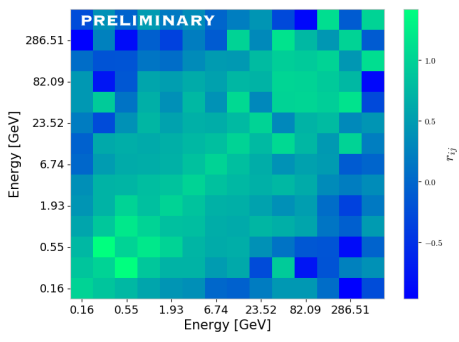

(c)

Figure 2: (a) red points: $C_{P}^{i j}$, gray points: $\sqrt{C_{P}^{i i}, C_{P}^{j j}}$, where bin $i$ is $0.5-1 \mathrm{GeV}$; (b) same as (a) but bin $i$ is $69.2-120.2$ $\mathrm{GeV}$; (c) cross-correlation coefficients matrix.

\section{Conclusions}

With respect to the previous auto-correlation analysis of the $\mathrm{UGRB}^{[1]}$, in the present work a general improvement in the data selection is mainly attributed to to the new event level analysis, Pass 8, which allows us to consider an extremely low background sample of events as well as to exclude the quartile of events with the worst containment angle. In addition to this, building an energy and flux dependent source mask contributes to avoid the leakage from resolved sources at low energy and to gain statistics at high energy. Also, defining an energy-dependent range of 


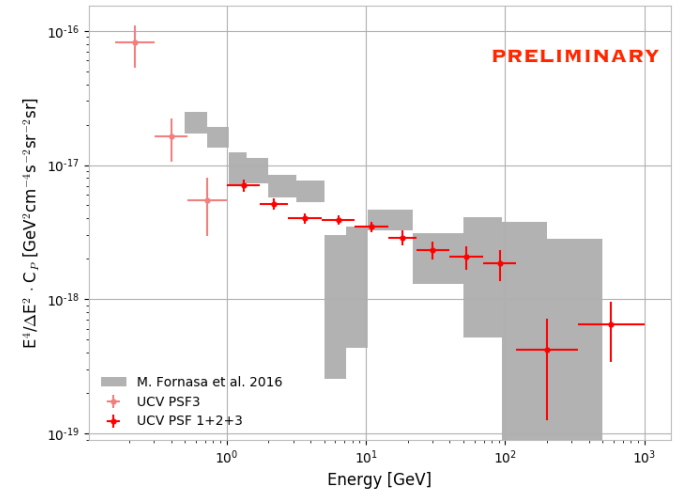

(a)

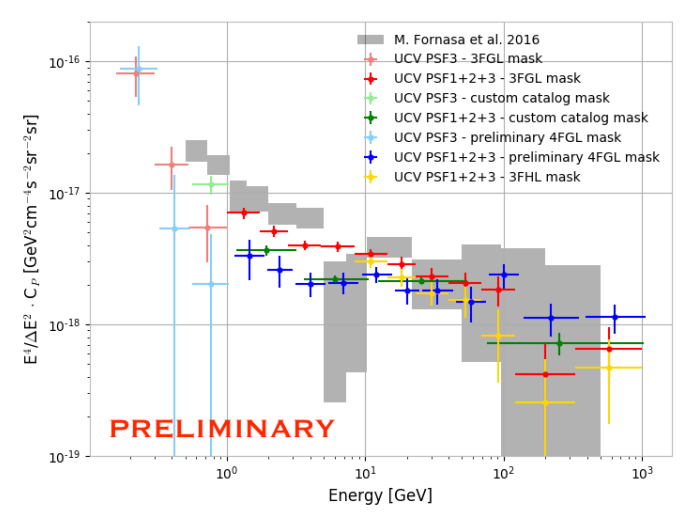

(b)

Figure 3: (a) Anisotropy energy spectrum of the UGRB; (b) Different anisotropy energy spectra of the UGRB obtained by masking different catalogs of sources

multipoles to estimate the level of anisotropy allows to reduce the impact of the large-scale Galactic emission contamination at low multipoles and from inaccurate PSF corrections at high multipoles. Auto- and cross-correlation analyses suggest that multiple populations of unresolved sources are contributing to the anisotropy signal, but a physical interpretation needs more and careful studies, first of all the dependence of the measurement on the masked catalog and hence on the instrument source detection efficiency.

\section{Acknoledgement}

The Fermi-LAT Collaboration acknowledges support for LAT development, operation and data analysis from NASA and DOE (United States), CEA/Irfu and IN2P3/CNRS (France), ASI and INFN (Italy), MEXT, KEK, and JAXA (Japan), and the K.A. Wallenberg Foundation, the Swedish Research Council and the National Space Board (Sweden).

\section{References}

[1] M. Fornasa et al., Angular power spectrum of the diffuse gamma-ray emission as measured by the Fermi Large Area Telescope and constraints on its dark matter interpretation, Phys. Rev. D 94, 123005 [astro-ph.HE]

[2] M. Ackermann et al., The spectrum of isotropic diffuse gamma-ray emission between $100 \mathrm{MeV}$ and $820 \mathrm{GeV}, \mathrm{ApJ}, 799,86$ [astro-ph.HE]

[3] M. Ajello et al., The origin of the extragalactic gamma-ray background and implications for the dark matter annihilation, ApJL, 800, L27 [astro-ph.HE]

[4] J.-Q. Xia et al., Tomography of the Fermi-LAT $\gamma$-ray diffuse extragalactic signal via cross correlations with galaxy catalogs, Astrophys.J.Suppl. 232 (2017) 10

[5] N. Fornengo et al., Evidence of cross-correlation between the CMB lensing and the gamma-ray sky, Astrophys.J. 802 (2015) no.1, L1 [astro-ph.CO] 
[6] E. Branchini et al., Cross-correlating the $\gamma$-ray sky with Catalogs of Galaxy Clusters, Astrophys.J.Suppl. 228 (2017) no.1, 8 [astro-ph.CO]

[7] S. Camera et al., Tomographic-spectral approach for dark matter detection in the cross-correlation between cosmic shear and diffuse $\gamma$-ray emission, JCAP 1506 (2015) no.06, 029 [astro-ph.CO]

[8] F. Acero et al., Fermi Large Area Telescope Third Source Catalog, Astrophys.J.Suppl. 218 (2015) no.2, 23 [astro-ph.HE]

[9] F. Acero et al., Development of the Model of Galactic Interstellar Emission for Standard Point-Source Analysis of Fermi Large Area Telescope Data, Astrophys.J.Suppl. 223 (2016) no.2, 26 [astro-ph.HE]

[10] M. Di Mauro et al., Deriving the contribution of blazars to the Fermi-LAT Extragalactic ?-ray background at E>10 GeV with efficiency corrections and photon statistics, arXiv:1711.03111 [astro-ph.HE]

[11] M. Ajello et al., 3FHL: The Third Catalog of Hard Fermi-LAT Sources, Astrophys.J.Suppl. 232 (2017) no.2, 18 [astro-ph.HE]

[12] http://www2.iap.fr/users/hivon/software/PolSpice/

[13] http://healpix.sourceforge.net/documentation.php 